\title{
Study of AZFc partial deletion gr/gr in fertile and infertile Japanese males
}

\author{
Cláudia Márcia Benedetto de Carvalho · Luciana Werneck Zuccherato • \\ Masato Fujisawa · Toshiro Shirakawa - Andrea Kely Campos Ribeiro-dos-Santos • \\ Sidney E. B. Santos · Sérgio Danilo Junho Pena • \\ Fabrício Rodrigues Santos
}

Received: 1 February 2006/ Accepted: 26 May 2006/Published online: 10 August 2006

(C) The Japan Society of Human Genetics and Springer-Verlag 2006

\begin{abstract}
A recurrent partial azoospermia factor C $(\mathrm{AZFc})$ deletion, called gr/gr, has been reported to be a male infertility risk factor. A specific type of $\mathrm{Y}$ chromosome observed in approximately $30 \%$ of Japanese males (haplogroup D derived at $\mathrm{YAP}+$ ) is believed to have a fixed gr/gr deletion. A recent study claimed that spermatogenic failure is more likely in males with D Y chromosomes, because of the gr/gr deletion, the presence of which is not well characterized among D haplogroup chromosomes. We therefore decided to perform a systematic study of the frequency of the gr/gr deletion in the Japanese. We studied fertile and infertile males to investigate the possibility of different $\mathrm{gr} / \mathrm{gr}$ frequencies. The deletions were detected by use of single tagged-sequences (STSs) and the $\mathrm{D}$ haplogroup sub-lineages typing were done by use
\end{abstract}

Electronic Supplementary Material Supplementary material is available to authorised users in the online version of this article at http://dx.doi.org/10.1007/s10038-006-0024-2.

C. M. B. de Carvalho - L. W. Zuccherato - S. D. J. Pena

Departamento de Bioquímica e Imunologia,

Universidade Federal de Minas Gerais,

Belo Horizonte, MG, Brazil

M. Fujisawa $\cdot$ T. Shirakawa

Kobe University Graduate School of Medicine, Kobe, Japan

A. K. C. Ribeiro-dos-Santos · S. E. B. Santos

Universidade Federal do Pará, Belém, PA, Brazil

F. R. Santos $(\bowtie)$

Departamento de Biologia Geral, ICB, UFMG,

Universidade Federal de Minas Gerais,

C.P. 486, Av. Antônio Carlos 6627,

31270-010 Belo Horizonte, MG, Brazil

e-mail: fsantos@icb.ufmg.br of the biallelic markers M174, M64, M116.1, 12f2.2, M15, M151, and M125. Analysis of gr/gr deleted Y chromosomes showed that all are classified as haplogroup D2, suggesting a lineage association. The subtype D2b1 was most frequent among the Japanese, in control and infertile samples. The haplogroups D2b2, $\mathrm{D}^{*}$, and $\mathrm{D} 1$ were not found in any population group. Remarkably, we observed no statistical difference between haplogroup D sub-lineages of the infertile and control groups, although the statistical power of this study is low. This study suggests lack of significant evidence of increased infertility risk in haplogroup D Japanese males. We were also able to establish the ancestral chromosome that suffered a gr/gr deletion, and propose a new $\mathrm{Y}$ chromosome phylogeny for haplogroup D and its derivatives. In summary, we were able to define the frequency of gr/gr deletion in Japanese males and show that the gr/gr deletion was probably present in the ancestral $\mathrm{Y}$ chromosome that entered Japan at least 12,000 years ago.

Keywords Y chromosome $\cdot$ Haplogroup D · $\mathrm{gr} / \mathrm{gr}$ deletion $\cdot$ Male infertility $\cdot \mathrm{AZFc}$

\section{Introduction}

Novel studies have revealed high molecular variability in the azoospermia factor $\mathrm{C}(\mathrm{AZFc})$ region among $\mathrm{Y}$ chromosomes of different haplogroups (Repping et al. 2006; reviewed by Carvalho and Santos 2005). This region, which spans $3.5 \mathrm{Mb}$ along the long arm of the $\mathrm{Y}$ chromosome, contains testis-specific genes involved in male spermatogenesis (Kuroda-Kawaguchi et al. 2001; Jobling and Tyler-Smith 2003). The genomic region has 
a complex structure full of palindromes and repeated segments prone to internal rearrangements (KurodaKawaguchi et al. 2001; Machev et al. 2004; Repping et al. 2003, 2006). Recombination of the direct repetitions b2/b4 results in complete deletion of AZFc region, causing spermatogenic failure in men (KurodaKawaguchi et al. 2001). Partial deletions also occur frequently $\left(3.8 \times 10^{-4}\right.$; Repping et al. 2006), producing a polymorphic Y chromosome (Jobling et al. 1996; Fernandes et al. 2004). A specific and recurrent partial $\mathrm{AZFc}$ deletion, called gr/gr, removes $1.6 \mathrm{Mb}$ of the $\mathrm{AZFc}$ and it has been identified as a risk factor in spermatogenic failure (Repping et al. 2003; de Llanos et al. 2005; Lynch et al. 2005; Ravel et al. 2006), although this has not been confirmed by other groups (Machev et al. 2004; Hucklenbroich et al. 2005; Carvalho et al. 2006). According to Repping et al. (2003) the D2b lineage, a D haplogroup sub-lineage particularly prevalent in Japan, should result in a risk of spermatogenic failure, because they would have the gr/ gr deletion fixed.

Remarkably, Kuroki et al. (1999) found fertile haplogroup D Japanese males with low sperm count. They suggested spermatogenic failure was more likely to occur among haplogroup D than among other $\mathrm{Y}$ chromosome types. Our group has previously published an association study of Japanese azoospermic and unknown fertility males (Carvalho et al. 2003) that did not support the findings of Kuroki et al (1999). In contrast with their results, we observed no statistical difference between $\mathrm{Y}$ chromosome haplotype frequencies in fertile and infertile males. However, the detailed characterization of the haplogroup D sub-lineages as well as the occurrence of the gr/gr deletion were not established in those samples. The current literature also provides few information about that issue (Repping et al. 2003). We therefore decided to investigate in detail the frequency of $\mathrm{gr} / \mathrm{gr}$ deletion in Japanese fertile and infertile males. We used our previously published fertile and infertile Japanese males (Carvalho et al. 2003) and typed for the new biallelic markers inside the D branch, in accordance with Jobling and Tyler-Smith (2003). The gr/gr deletion was analyzed in both fertile and infertile groups using the same single-tagged sequence (STS) markers proposed by Repping et al. (2003).

This study shows that all gr/gr deleted chromosomes found in Japanese samples can be classified as haplogroup D2, suggesting a founder effect. The subtype D2b1 was most frequent among the Japanese, both in control and infertile samples. Some haplogroups, $\mathrm{D} 2 \mathrm{~b} 2, \mathrm{D}^{*}$, and $\mathrm{D} 1$, were not found in any population group and no significant association of haplogroup D with increased risk of infertility was observed in this study. We were also able to establish the ancestral chromosome that suffered a gr/gr deletion, and propose a new Y chromosome phylogeny for haplogroup $\mathrm{D}$ and its derivatives. In summary, we were able to define the frequency of $\mathrm{gr} / \mathrm{gr}$ deletion in Japanese males and showed that the gr/gr deletion was probably already present among the ancestral $\mathrm{Y}$ chromosome population that entered Japan at least 12,000 years ago.

\section{Materials and methods}

The Japanese subjects are described elsewhere (Fujisawa et al. 2001; Carvalho et al. 2003). Briefly, we studied 78 infertile males, and 56 males of unknown fertility as control. The infertile males were subdivided into two groups-49 patients were classified as azoospermic (Sertoli cell only, severe hypospermatogenesis, maturation arrest) on the basis of clinical and biopsy data and 29 were classified generally as infertile because they did not provide biopsy data. These samples had previously been tested for the occurrence of AZFa, b, and c deletions (Carvalho et al. 2003). All samples were submitted to molecular analysis of $\mathrm{Y}$ chromosome gr/gr deletion in accordance with Repping et al. (2003). First, we screened samples for the presence or absence of the STS markers sY1291 and 50f2/C. Both define the partial deletions commonly found in the $\mathrm{Y}$ chromosome, which are $\mathrm{gr} / \mathrm{gr}, \mathrm{b} 1 / \mathrm{b} 3$ (Repping et al. 2003), and b2/b3 (Fernandes et al. 2004; Repping et al. 2004). The polymorphic deletion markers 50f2/C or DYS7/C (Genbank accession number Y07728) and sY1191 lie in the proximally located u3 block. In a second round, the individuals with deletions were tested by STS typing: sY1161, sY1191, sY1206, sY1201, and sY1258. All STSs primers were used at $0.5 \mu \mathrm{mol} \mathrm{L}{ }^{-1}$ concentration for PCR. To avoid false negative results we performed all STS PCR assays in duplex format containing primers for the Yp marker SRY+465 (Shinka et al. 1999) at $0.25 \mu \mathrm{mol} \mathrm{L}^{-1}$. Results were always repeated to confirmation. The primer sequences for STSs are available from GenBank under the accession numbers: sY1161, G66148; sY1191, G73809; sY1291, G72340; sY1206, G68331; sY1201, G67170; and sY1258, G75479. The procedure for detection of the 50f2/C polymorphic deletion has been described elsewhere (Jobling et al. 1996). The PCR products were resolved in $6 \%$ polyacrylamide gels and silver-stained in accordance with Santos et al. (1996). 
All samples had previously been assigned to the $\mathrm{Y}$ chromosome haplogroups (Carvalho et al. 2003). In this study haplogroup D (defined by the presence of the Alu insertion, YAP+) samples were also typed by minisequencing (Carvalho and Pena 2005) using the single nucleotide polymorphisms (SNPs) M174, M64, M116.1, M151, and M125. We used the nomenclature proposed by Jobling and Tyler-Smith (2003). Other insertion/deletion polymorphisms M15 and $12 \mathrm{f} 2.2$ were typed using $6 \%$ polyacrylamide gel as described above. The procedure for analysis of the $12 \mathrm{f} 2.2$ marker is described elsewhere (Rosser et al. 2000). The primers used to detect the M15 insertion/deletion and other SNP markers are reported in the supplementary information. Statistical tests were performed using Arlequin ver. 2.0 software (Schneider et al. 2000). Specifically, we used an exact test of population differentiation to verify whether haplogroup frequencies were statistically different among groups. We also used AMOVA (population genetic structure inferred by analysis of variance) to quantify the distribution of variation between and within fertile and infertile groups. We used the PS power and sample-size program of Dupont and Plummer (Version 1.0.17; freely available at: http:// www.mc.vanderbilt.edu/prevmed/ps.htm) for samplesize estimation.

\section{Results}

Among 134 Japanese males, 41 had gr/gr deleted Y chromosomes: 19 control, 11 azoospermic, and 11 infertile males without biopsy data (Table 1). Using an exact test of population differentiation, the presence of gr/gr deletions was compared among azoospermic, infertile without biopsy data, and control males $(P=0.26235 \pm 0.01458)$. The exact test revealed no statistically significant difference at the $5 \%$ level for all groups.

We also observed other kinds of partial AZFc deletion in both groups. We did not use sY1197 and sY142 (Repping et al. 2003, 2004) to identify b2/b3 and b1/b3 deletions, but analysis of the Y chromosome map indicates that sY1258 can provide the same information. We identified four b2/b3-deleted samples, two in the control and two in the infertile group (Table 1). All were typed as haplogroup $\mathrm{N}$, which have the $\mathrm{b} 2 / \mathrm{b} 3$ deletion fixed (Fernandes et al. 2004; Repping et al. 2004). The b1/b3 deletion was detected in one control sample (Table 1). None of these is believed to be associated with spermatogenic failure.

Interestingly, a new type of deletion was detected in this study and is marked by the absence of STS sY1191, 50f2/C, and sY1258. This deletion has not previously been observed when using these STS markers. We

Table 1 Y chromosome deletions detected in Japanese infertile (with and without biopsy data) and control males. Infertile samples with the biopsy data are described in detail

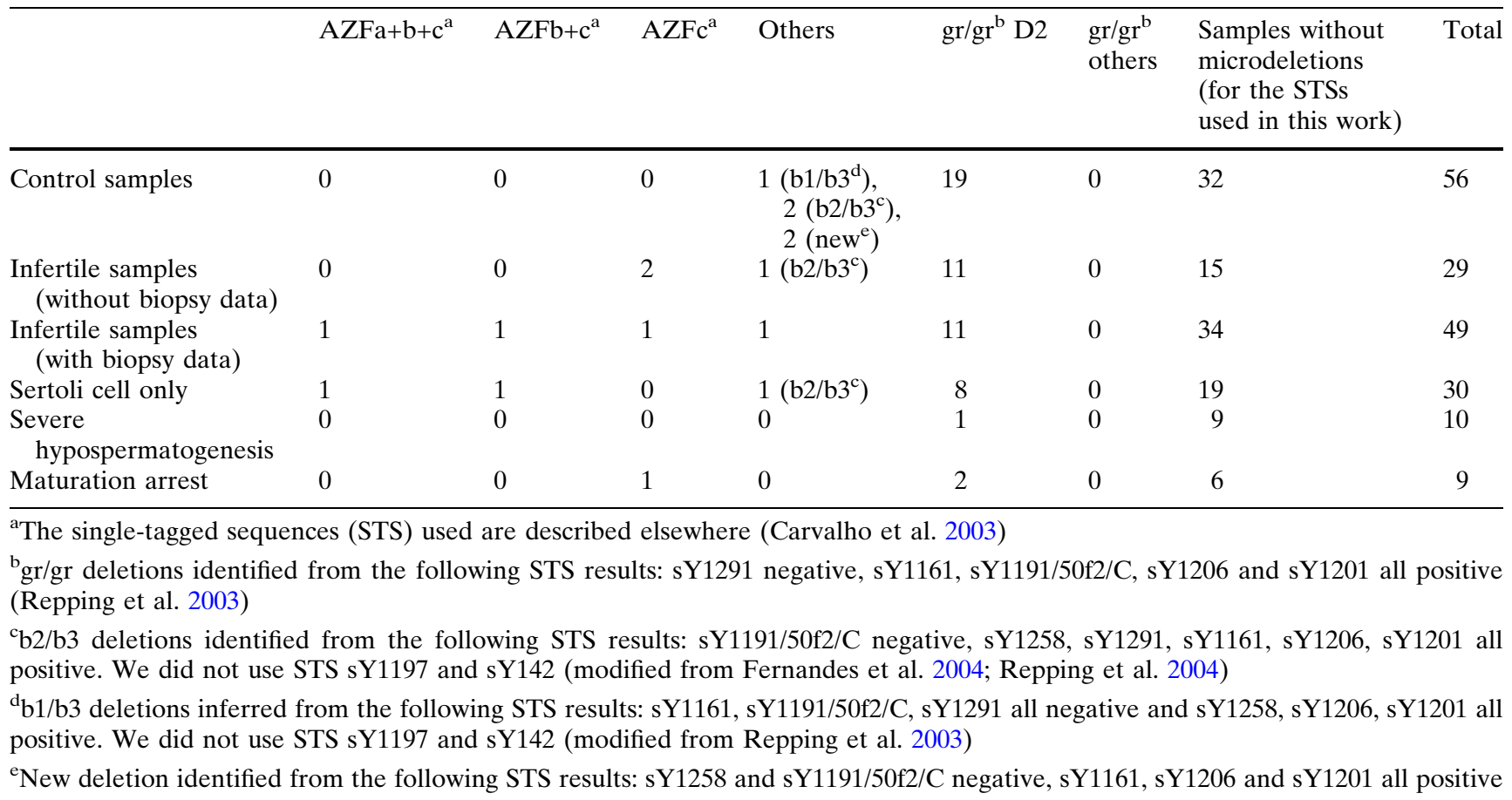


detected two control samples with this new deletion, both typed as $\mathrm{Y}$ chromosome haplogroup $\mathrm{C}$ and bearing an atypical $\alpha \mathrm{hI}$ polymorphism type (Carvalho et al. 2003). Using several probes annealing to Yp and Yq (including 50f2), Jobling et al. (1996) reported that at least two different deletion events, both involving the absence of 50f $2 / \mathrm{C}$ band, could be observed for haplogroup $\mathrm{C}$ chromosomes. The most frequent one (nine out of ten deleted individuals) was called "large", because it co-deleted bands from other probes present at Yq11. The 50f2/C "large" deletion has been detected in several populations, including Mongolian and Japanese. We therefore speculate that the 50f2/C "large" polymorphism found in haplogroup C males using southern blot (Jobling et al. 1996) is probably the same as the deletion detected by us using STS sY1191, 50f2/C, and sY1258.

Analysis of gr/gr deleted $\mathrm{Y}$ chromosomes revealed that all were classified as haplogroup D. The biallelic markers M15, M174, M116.1, M64, M125, M151, and $12 \mathrm{f} 2.2$ were used to resolve the $\mathrm{D}$ branch in both control and infertile samples. The haplotyping results can be seen in Table 2 and in the phylogenetic tree in Fig. 1 . We observed that all Japanese D males $(n=41)$ also belong to the lineage D2 (M174 and M64-derived alleles and gr/gr deletion). According to our haplotyping results (Fig. 1), the D2 lineage could be further divided into D2b* (defined as derived allele at M116.1 and ancestral at M125, 12f2.2, and M151), D2b1 (defined as derived alleles at M116.1, M125, and $12 \mathrm{f} 2.2$ and ancestral at M151) , and D2b2 (defined as derived alleles at M116.1 and M151 and ancestral at M125 and 12f2.2). The frequency was highest for D2b1, both in control (53\%) and infertile samples (73\%), and D2b* was present in $37 \%$ of control individuals and in $18 \%$ of infertile males. The frequency of D2* (defined as derived at M64, and ancestral at M116.1, M151, M125, and $12 \mathrm{f} 2.2$ ) was about $9-10 \%$ in both groups. Haplogroups $\mathrm{D} 2 \mathrm{~b} 2, \mathrm{D}^{*}$, and $\mathrm{D} 1$ were not found in any

Table 2 Frequency distribution of infertile and control samples from the D branch. All samples are gr/gr deleted

\begin{tabular}{lcll}
\hline $\begin{array}{l}\text { Haplogroups } \\
\text { 22 branch }\end{array}$ & $\begin{array}{l}\text { Control } \\
\text { samples, } \\
N=19\end{array}$ & $\begin{array}{l}\text { Infertile samples } \\
\text { (azoospermic), } \\
N=11\end{array}$ & $\begin{array}{l}\text { Infertile samples } \\
\text { (without biopsy } \\
\text { data), } N=11\end{array}$ \\
\hline D* & 0 & 0 & 0 \\
D1 & 0 & 0 & 0 \\
D2* & 2 & 2 & 0 \\
D2b* & 7 & 1 & 3 \\
D2b1 & 10 & 8 & 8 \\
D2b2 & 0 & 0 & 0
\end{tabular}

$P=0.3955 \pm 0.0129$ using the exact test of population differentiation (Schneider et al. 2000)

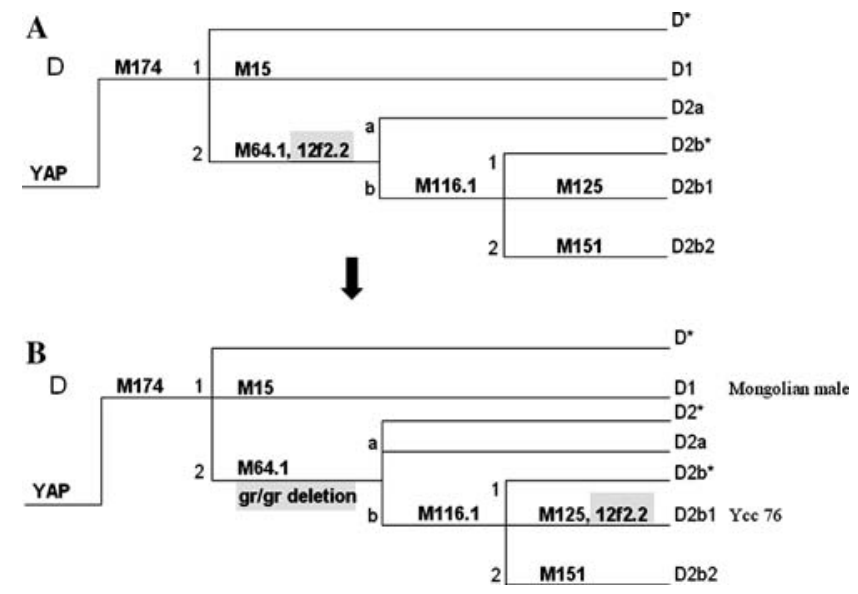

Fig. 1 Detailed D branch phylogeny of the human Y chromosome tree. The biallelic markers specified were tested in all control and infertile males. a D branch according to Jobling and Tyler-Smith (2003)*. b D branch proposed by this study. The markers altered are highlighted in the figure. The designations for the branches are based on Jobling and Tyler-Smith (2003). *Although we have not tested markers to detect the D2a haplogroup specifically, we depict it here to show the discrepancies between the previous published YCC tree and the most likely tree according to our data

population group (Fig. 1). The haplogroup frequencies were compared among control, azoospermic, and infertile males without biopsy data using the exact test of population differentiation $(P=0.39550 \pm 0.01292)$. The test revealed no statistically significant differences at the 5\% level for all group comparisons. The AMOVA results also indicated that $100 \%$ of the variation was found within the population, i.e. no differentiation could be attributed to a comparison of $\mathrm{Y}$ the chromosomes of control and infertile patients. That we did not find an association between the presence of the $\mathrm{gr} /$ gr deletion in $\mathrm{Y}$ chromosomes and the infertile phenotype in Japanese males might be because of the small sample size of infertile males. To achieve a significance level of 0.05 with a statistical power of $80 \%$, a sample size of over 1,274 well-characterized patients would have been required. Replication studies in larger cohorts, and functional studies, are needed.

To define when the gr/gr deletion event occurred along the D lineage, we analyzed all haplogroup D subdivisions. A haplogroup D1 Mongolian male (M15 derived) does not have the gr/gr deletion, suggesting that the deletion appears only in chromosomes of the M64-derived branch, the origin of the D2 lineage. On the basis of these results, a new organization of the $\mathrm{D}$ branch was proposed (Fig. 1). This also changes the current phylogeny (The Y Chromosome Consortium 2002; Jobling and Tyler-Smith 2003) concerning the position of the $12 \mathrm{f} 2.2$ mutation. According to our 
analysis of haplogroup D and its derived sublineages, the $12 \mathrm{f} 2.2$ deletion occurred more recently in an ancestral chromosome of the D2b1 lineage. We have also used a sample (YCC-76) from the Y Chromosome Consortium, which has been re-classified in the new tree (Fig. 1). Although we have not typed P42, this sample has the derived state in the marker M125, which defines the lineage D2b1, the most common in Japan. This confirms a previous doubt about the haplogroup classification of YCC-76 male that should bear a derived state in M125 (F.R. Santos, personal data).

\section{Discussion}

Although this work showed that the gr/gr deletion is fixed in D2b haplogroup, as suggested by Repping et al. (2003), we also showed it occurred in an older ancestral $\mathrm{Y}$ chromosome lineage, which is $\mathrm{D} 2$. Approximately $83-88 \%$ of D Japanese males can be classified into clade D2b and $100 \%$ into D2 (Table 2). The D2 chromosomes are mainly (if not exclusively) found in Japanese males, in which its frequency of occurrence is high. A hypothetical selection against D2b gr/gr deleted chromosomes (Repping et al. 2003) can hardly explain the high frequency of this chromosome in Japan. As proposed by Underhill et al. (2001) and Hammer et al. (2006), genetic drift is the most likely factor underlying the distribution of haplogroup D (including derived ones) in Asia. Molecular studies have proposed that haplogroup D entered Japan approximately 12,000-20,000 years ago from central Asia (Hammer et al. 2006; Katoh et al. 2005), probably brought by the Jomon, the first colonizers of the Japanese islands. Among the ancestral Jomon Y chromosomes there was probably a D2 haplogroup carrier. The gr/gr deletion present in D2 chromosomes could have originated in an ancestral male before or just after the migration to Japan. Thus, it seems the gr/gr deletion found in D2 chromosomes has not restricted the spread of the chromosome. D2 chromosome frequencies are highly variable, depending on the island or the population analyzed (Tajima et al. 2002): Ainu (from Hokkaido: $87.5 \%$ ), Honshu (36.6\%), Kyushu (28\%), and Okinawa $(55.6 \%)$. D2 is, however, the most representative haplogroup found in Japan.

Other clues also indicate that the sub-lineage D2 (defined as M64-derived and the gr/gr deletion) is found exclusively in Japanese males. The D clade is rare in Asian populations other than in Japan and Tibet (Underhill et al. 2000, 2001; Tajima et al. 2002; Jin et al. 2003; Deng et al. 2004; Katoh et al. 2005; Hammer et al. 2006). Available data also indicate that
D*( $\times$ D2), but not D2, occurs in Tibet (Deng et al. 2004; Hammer et al. 2006). The population differences found in Japan can be attributed to another important migration wave which occurred $\sim 2,300$ years ago, bringing the Yayoi people from the Korean peninsula, carrying $\mathrm{Y}$ lineages like $\mathrm{O}$, but not $\mathrm{D}$ lineages. The admixture hypothesis proposed by Hammer and Horai (1995) suggested that modern Japanese people are derived from both Jomon and Yayoi people. The haplotype differences observed among the islands would probably reflect the impact of this later migration. Thus, even after the more recent arrival of Yayoi people, who also brought the language spoken today, D2 Y chromosomes still remain in the Japanese population at high frequency, despite the occurrence of intensive gene flow bringing non-gr/gr deleted Ychromosomes. We suggest that possible differentiation observed among Japanese populations, or fertile/ infertile comparisons, can be more easily explained by drift, population substructure, and differential gene flow. Taken together, our results suggest a lack of significant evidence associating the presence of the $\mathrm{gr} /$ gr deletion in $\mathrm{Y}$ chromosomes and the infertile phenotype in Japanese males.

Unexpectedly we discovered a different organization of the D2 sub-lineage tree. The previous tree suggests the $12 \mathrm{f} 2.2$ deletion as a marker of the D2 lineage; we showed here this is not correct. The Y Chromosome Consortium (2002) organized the phylogenetic $\mathrm{Y}$ chromosome tree using information provided by several research groups, many times using different markers. Thus, some nodes in the tree are not well resolved, so fine scale revision is required, as we did for D2 (Fig. 1). It is important to re-consider the haplotyping of the YCC sample panel in the light of the new revisions, as we did for the YCC-76 sample (Fig. 1). It was re-classified as D2b1 haplogroup instead of $\mathrm{D} 2 \mathrm{a}$, as previously reported. All related markers should be retyped, however, to confirm the actual shape of the tree around the D haplogroup.

Acknowledgments We would like to thank anonymous sample donors, who enabled this work to be performed. We also thank Neuza Antunes, Kátia Barroso, and Míriam Rodrigues for technical assistance. This work was supported by the Brazilian agency CNPq.

\section{References}

Carvalho CMB, Fujisawa M, Shirakawa T, Gotoh A, Kamidono S, Paulo TF, Santos SEB, Rocha J, Pena SDJ, Santos FR (2003) Lack of association between Y chromosome haplogroups and male infertility in Japanese men. Am J Med Genet 116A:152-158 
Carvalho CMB, Santos FR (2005) Human Y-chromosome variation and male dysfunction. $\mathrm{J}$ Mol Genet Med 1:63-75

Carvalho CMB, Pena SDJ (2005) Optimization of a multiplex minisequencing protocol for population studies and medical genetics. Genet Mol Res 4:115-125

Carvalho CM, Zuccherato LW, Bastos-Rodrigues L, Santos FR, Pena SD (2006) No association found between gr/gr deletions and infertility in Brazilian males. Mol Hum Reprod 12:269-283

Deng W, Shi B, He X, Zhang Z, Xu J, Li B, Yang J, Ling L, Dai C, Qiang B, Shen Y, Chen R (2004) Evolution and migration history of the Chinese population inferred from Chinese Y-chromosome evidence. J Hum Genet 49:339-348

Fernandes S, Paracchini S, Meyer LH, Floridia G, Tyler-Smith C, Vogt PH (2004) A large AZFc deletion removes DAZ3/ DAZ4 and nearby genes from men in Y haplogroup N. Am J Hum Genet 74:180-187

Fujisawa M, Shirakawa T, Kanzaki M, Okada H, Arakawa S, Kamidono S (2001) Y-chromosome microdeletion and phenotype in cytogenetically normal men with idiopathic azoospermia. Fertil Steril 76:491-495

Hammer MF, Horai S (1995) Y chromosomal DNA variation and the peopling of Japan. Am J Hum Genet 56:951-962

Hammer MF, Karafet TM, Park H, Omoto K, Harihara S, Stoneking M, Horai S (2006) Dual origins of the Japanese: common ground for hunter-gatherer and farmer $\mathrm{Y}$ chromosomes. J Hum Genet 51:47-58

Hucklenbroich K, Gromoll J, Heinrich M, Hohoff C, Nieschlag E, Simoni M (2005) Partial deletions in the AZFc region of the $\mathrm{Y}$ chromosome occur in men with impaired as well as normal spermatogenesis. Hum Reprod 20:191-197

Jin HJ, Kwak KD, Hammer MF, Nakahori Y, Shinka T, Lee JW, Jin F, Jia X, Tyler-Smith C, Kim W (2003) Y-chromosomal DNA haplogroups and their implications for the dual origins of the Koreans. Hum Genet 114:27-35

Jobling MA, Tyler-Smith C (2003) The human Y chromosome: an evolutionary marker comes of age. Nat Rev Genet 4:598612

Jobling MA, Samara V, Pandya A, Fretwell N, Bernasconi B, Mitchell RJ, Gerelsaikhan T, Dashnyam B, Sajantila A, Salo PJ (1996) Recurrent duplication and deletion polymorphisms on the long arm of the Y chromosome in normal males. Hum Mol Genet 5:1767-1775

Katoh T, Munkhbat B, Tounai K, Mano S, Ando H, Oyungerel G, Chae GT, Han H, Jia GJ, Tokunaga K, Munkhtuvshin N, Tamiya G, Inoko H (2005) Genetic features of Mongolian ethnic groups revealed by Y-chromosomal analysis. Gene 346:63-70

Kuroda-Kawaguchi T, Skaletsky H, Brown LG, Minx PJ, Cordum HS, Waterston RH, Wilson RK, Silber S, Oates R, Rozen S, Page DC (2001) The AZFc region of the $Y$ chromosome features massive palindromes and uniform recurrent deletions in infertile men. Nat Genet 29:279-286

Kuroki Y, Iwamoto T, Lee J, Yoshiike M, Nozawa S, Nishida T, Ewis AA, Nakamura H, Toda T, Tokunaga K, Kotliarova SE, Kondoh N, Koh E, Namiki M, Shinka T, Nakahori Y (1999) Spermatogenic ability is different among males in different Y chromosome lineage. J Hum Genet 44:289-292

de Llanos M, Ballesca JL, Gazquez C, Margarit E, Oliva R (2005) High frequency of gr/gr chromosome Y deletions in consecutive oligospermic ICSI candidates. Hum Reprod 20:216-220

Lynch M, Cram DS, Reilly A, O'Bryan MK, Baker HW, de Kretser DM, McLachlan RI (2005) The Y chromosome gr/ gr subdeletion is associated with male infertility. Mol Hum Reprod 11:507-512
Machev N, Saut N, Longepied G, Terriou P, Navarro A, Levy N, Guichaoua M, Metzler-Guillemain C, Collignon P, Frances AM, Belougne J, Clemente E, Chiaroni J, Chevillard C, Durand C, Ducourneau A, Pech N, McElreavey K, Mattei MG, Mitchell MJ (2004) Sequence family variant loss from the AZFc interval of the human Y chromosome; but not gene copy loss; is strongly associated with male infertility. J Med Genet 41:814-825

Ravel C, Chantot-Bastaraud S, El Houate B, Mandelbaum J, Siffroi JP, McElreavey K (2006) GR/GR deletions within the azoospermia factor $\mathrm{c}$ region on the $\mathrm{Y}$ chromosome might not be associated with spermatogenic failure. Fertil Steril 85:229-231

Repping S, Skaletsky H, Brown L, van Daalen SK, Korver CM, Pyntikova T, Kuroda-Kawaguchi T, de Vries JW, Oates RD, Silber S, van der Veen F, Page DC, Rozen S (2003) Polymorphism for a $1.6-\mathrm{Mb}$ deletion of the human $\mathrm{Y}$ chromosome persists through balance between recurrent mutation and haploid selection. Nat Genet 35:247-251

Repping S, van Daalen SK, Korver CM, Brown LG, Marszalek JD, Gianotten J, Oates RD, Silber S, van der Veen F, Page DC, Rozen S (2004) A family of human Y chromosomes has dispersed throughout northern Eurasia despite a 1.8-Mb deletion in the azoospermia factor c region. Genomics 83:1046-1052

Repping S, van Daalen SK, Brown LG, Korver CM, Lange J, Marszalek JD, Pyntikova T, van der Veen F, Skaletsky H, Page DC, Rozen S (2006) High mutation rates have driven extensive structural polymorphism among human Y chromosomes. Nat Genet 38:463-467

Rosser Z, Zerjal T, Hurles ME, Adojaan M, Alavantic D, Amorim A, Amos W et al (2000) Y-chromosomal diversity within Europe is clinal and influenced primarily by geography rather than language. Am J Hum Genet 67:1526-1543

Santos FR, Bianchi NO, Pena SD (1996) Worldwide distribution of human Y-chromosome haplotypes. Genome Res 6:601611

Shinka T, Tomita K, Toda T, Kotliarova SE, Lee J, Kuroki Y, Jin DK, Tokunaga K, Nakamura H, Nakahori Y (1999) Genetic variations on the $\mathrm{Y}$ chromosome in the Japanese population and implications for modern human $\mathrm{Y}$ chromosome lineage. J Hum Genet 44:240-245

Suchneider S, Roessli D, Excoffier L (2000) Arlequin ver. 2.000: a software for population genetic data analysis. Genetic and Biometry Laboratory, University of Geneva, Geneva, Switzerland

Tajima A, Pan IH, Fucharoen G, Fucharoen S, Matsuo M, Tokunaga K, Juji T, Hayami M, Omoto K, Horai S (2002) Three major lineages of Asian Y chromosomes: implications for the peopling of east and southeast Asia. Hum Genet 110:80-88

The Y Chromosome Consortium (2002) A nomenclature for the tree of human Y-chromosomal binary haplogroups. Genome Res 12:339-348

Underhill PA, Shen P, Lin AA, Jin L, Passarino G, Yang WH, Kauffman E, Bonne-Tamir B, Bertranpetit J, Francalacci P, Ibrahim M, Jenkins T, Kidd JR, Mehdi SQ, Seielstad MT, Wells RS, Piazza A, Davis RW, Feldman MW, CavalliSforza LL, Oefner PJ (2000) Y chromosome sequence variation and the history of human populations. Nat Genet 26:358-361

Underhill PA, Passarino G, Lin AA, Shen P, Mirazon Lahr M, Foley RA, Oefner PJ, Cavalli-Sforza LL (2001) The phylogeography of $\mathrm{Y}$ chromosome binary haplotypes and the origins of modern human populations. Ann Hum Genet 65:43-62 